\title{
Linear momentum increase and negative optical forces at dielectric interface
}

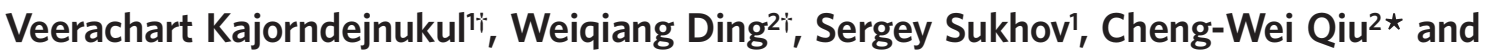 \\ Aristide Dogariu1*
}

Light carries momenta that can be transferred to objects. Relying on gradient forces created by structured light, one can trap and move microscopic particles. Aside from the conservative action of gradient forces, light always pushes an object along its direction of propagation. Here, we demonstrate that gradientless light fields can exert pulling forces on arbitrary objects in a purely passive dielectric environment and without resorting to non-paraxial illumination, interference of multiple beams, gain or other exotic materials. The forces acting against the flow of light arise naturally due to the appropriate amplification of the photon linear momentum when light is scattered from one dielectric medium into another with higher refractive index. This situation opens up a number of intriguing prospects for optical forces and their effects on surface-bound objects. Here, we demonstrate that this new mechanism can be used to manipulate objects over macroscopic distances along dielectric interfaces.

Recently, so-called optical 'tractor beams' have attracted a great deal of interest, largely because of their intriguing potential for micromanipulation ${ }^{1-9}$. A tractor beam per se is gradientless in intensity along the propagation direction, but it can exert a 'negative' force that pulls the scatterer in a direction opposite to the propagation of light, in contrast to the usually 'positive' push exerted by radiation pressure. In addition, this negative force is non-conservative, acts over longer distances, and may offer new possibilities for optical manipulation that differ from optical gradient-based tweezers $^{10-12}$ or other nano-optomechanical systems ${ }^{13,14}$.

For a negative force to occur, the fundamental requirement is that, after interaction with an object, the momentum of light should intensify along the direction of the incident beam, and different approaches have been proposed to achieve this ${ }^{9}$. One simple way makes use of gain material to amplify the momentum of the outgoing light ${ }^{4}$. Appropriate conditions for a negative force can also be generated when multipoles are excited simultaneously inside a subwavelength spherical scattere ${ }^{2,3}$. By increasing the forward scattering from objects that are highly scattering or have simple geometrical shapes, one can generate a negative force even with much larger objects ${ }^{7}$. Unfortunately, these approaches require a non-paraxial incident beam, which limits the spatial regions where strictly negative forces may occur ${ }^{1-3,7,8}$.

In this Letter, we demonstrate that stable negative forces are possible even when using ordinary, unstructured paraxial beams. Moreover, we show that a negative force can be created without any exotic properties of gain or left-handed media. Instead, our approach is based on the universal characteristics of optical forces acting on objects moving at the interface between different dielectric media.
One of the hypothetical realizations of a tractor beam discussed in the literature considers the question about the magnitude of electromagnetic momentum in dielectric media, that is, the AbrahamMinkowski controversy ${ }^{15,16}$. According to Minkowski's approach, the momentum of a photon increases $n$ times upon entering a dielectric medium $\left(p_{\mathrm{M}}=n \hbar \omega / c\right.$, where $n$ is the refractive index of the medium $)^{17}$. When illuminated by an optical pulse from a medium with lower refractive index, a dielectric object is therefore expected to 'jump' towards the source of light ${ }^{15,16}$. However, this simplified description does not take into account the deformability of the object and also mishandles the momentum associated with the material body ${ }^{15}$. As discussed by Balazs ${ }^{18}$ and Brevik ${ }^{19}$, a perfectly transmitting dielectric of higher refractive index than its surroundings will in fact advance in the direction of propagation of the wave packet. Nevertheless, both theoretical considerations ${ }^{20,21}$ and experiments reported so far ${ }^{16,22,23}$ indicate that under steady illumination the optical force acting on objects immersed or embedded in dispersionless dielectric media can be described in terms of the transfer of Minkowski momentum. In the following we will discuss how this can be used to create negative forces.

Let us examine the situation described in Fig. 1a. A scatterer with refractive index $n_{3}$ is placed at the interface of two media (for example, a gas and a liquid) with refractive indices $n_{1}$ and $n_{2}>n_{1}$. The scatterer is illuminated by a beam of light (plane wave) such that some of the incident and scattered rays lie in different media, as shown in Fig. 1a. In the conditions depicted here, both the direction and the magnitude of the momentum $\left(\mathbf{p}_{\mathrm{M}}\right)$ of the light change. According to Minkowski's approach, when going from medium 1 to medium 2, the momentum increases; consequently, the scattering force, in particular the component of the force experienced by the object along the interface, $F_{x} \propto-\Delta p_{\mathrm{M}, x}=(\hbar \omega / c)\left(n_{1} \cos \theta_{1}-n_{2} \cos \theta_{2}\right)$, may become negative so that the total momentum is conserved. A complete analysis of all force components is given in Supplementary Section S2. Because the relation between $\theta_{1}$ and $\theta_{2}$ depends on the shape and optical properties of the system, there can be situations when the scatterer moves in a direction opposite to the incident wave. In this scheme, it is the presence of the medium with high refractive index that provides the increase in forward momentum, even in a passive environment. Thus, according to the classification scheme discussed in ref. 9, the situation can be described as a 'tractor beam' action that is assisted by the environment surrounding the object.

We note that this increase in the forward momentum depends on both reflection and refraction in the medium with higher refractive index. This is why, according to Minkowski's description, negative force would not occur, for example, for plane-parallel objects. Indeed, the angles for such an object are related according to

'CREOL, The College of Optics and Photonics, University of Central Florida, 4000 Central Florida Boulevard, Orlando, Florida 32816 , USA, ${ }^{2}$ Department of Electrical and Computer Engineering, National University of Singapore, 4 Engineering Drive 3, Singapore 117576; ${ }^{\top}$ These authors contributed equally to this work. ^e-mail: chengwei.qiu@nus.edu.sg; adogariu@creol.ucf.edu 

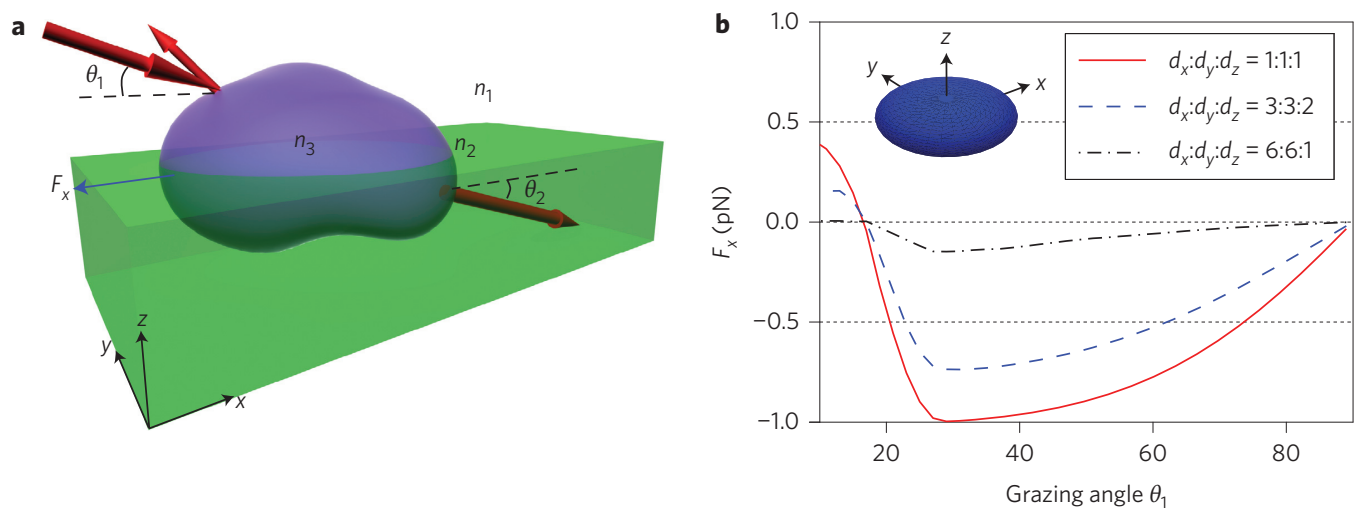

Figure 1 | Forward momentum amplification and backward particle motion when a ray propagates from air into water through the scatterer. $\mathbf{a}$, Schematic of the forward momentum amplification. Red arrows represent directional vectors along the incident, reflected and transmitted rays. The lengths of the directional vectors are proportional to the refractive index of the medium in which the rays lie. As a result of momentum conservation, a negative scattering force $F_{x}$ (blue arrow) is exerted on the scatterer along the interface. $\mathbf{b}$, The $x$-component of optical force as a function of grazing angle $\theta_{1}$ acting on spheroids with different main axes $d_{x}, d_{y}$ and $d_{z}$ (see inset) with the cross-sectional area along the interface remaining the same $\left(d_{x}=d_{y}=10 \mu \mathrm{m}\right)$. Forces are calculated with a ray-tracing technique for spheroids with refractive index $n_{3}=1.42$ floating at the air-water interface under the illumination of a $p$-polarized plane wave with irradiance of $10 \mu \mathrm{W} \mu \mathrm{m}^{-2}$

Snell's law $\left(n_{1} \cos \theta_{1}=n_{2} \cos \theta_{2}\right)$ and the force along the surface is $F_{x}=(P / c)\left(n_{1} \cos \theta_{1}-R n_{1} \cos \theta_{1}-T n_{2} \cos \theta_{2}\right) \equiv 0$, where $P$ is the optical power incident onto the plane-parallel object characterized by the intensity reflection and transmission coefficients $R$ and $T$. For objects with arbitrary shapes, a more detailed analysis is necessary to establish the detailed conditions for which negative force can be generated. As one can see from Fig. 1b, the occurrence of negative force is a rather general phenomenon, but, of course, non-absorbing dielectric objects of different shapes could experience negative forces of different strengths. For instance, Fig. 1b illustrates a few examples of ray-tracing calculations of scattering forces acting on objects with simple shapes (spheroids). One can clearly see that, in accordance to the discussion above, the flatter the shape of a spheroid, the smaller the negative force acting on it. Of course, at normal incidence $\left(\theta_{1}=\right.$ $\left.90^{\circ}\right)$ there will be no component of negative force along the surface for circularly symmetric objects (Fig. 1b).

To verify the concept of surface-assisted negative force, we performed a systematic experiment using small drops of dodecane (refractive index, 1.421; diameter, $\sim 10 \mu \mathrm{m}$ ) formed at the water surface (see Methods for a detailed description of the preparation procedure). The small floating droplets were illuminated at a grazing angle of $25^{\circ}$ by a loosely focused Gaussian laser beam (power, $1.2 \mathrm{~W}$; diameter, $\sim 300 \mu \mathrm{m})$. Under these conditions, the irradiance in the vicinity of the centre of the illumination spot $\left(\sim 35 \mu \mathrm{W} \mu \mathrm{m}^{-2}\right)$ was three orders of magnitude smaller than typical irradiances used in optical tweezers ${ }^{11}$ and two orders of magnitude lower than in a recent experimental realization of a tractor beam $^{9}$. This irradiance is also small enough to leave the water surface unperturbed, in contrast to the experiments reported in ref. 24. The movement of microdroplets was continuously imaged with a microscope and recorded with a charge-coupled device (CCD) camera. The particles' trajectories were subsequently analysed using particle-tracking techniques, as described in the Methods.

An example of reconstructed trajectories of floating particles is shown in Fig. 2a. As can be clearly seen, the droplets that happen to be inside the illumination spot manifest a distinct directional motion against the beam propagation. On the other hand, particles outside the illumination spot follow different directions of motion, with trajectories influenced to a certain degree by residual macroscopic flows on the water surface and, to a minor extent, by Brownian forces. As can be seen from Fig. 2a, negative motion of particles is observed over distances of $\sim 200 \mu \mathrm{m}$. In principle, the particles' movement can be affected by several mechanisms: gradient and scattering optical forces, drag forces and Brownian forces. At the working wavelength of $532 \mathrm{~nm}$, dodecane and water are essentially non-absorbing and direct thermally induced forces are not expected to make a significant contribution (Supplementary Section S6 provides an estimate of the influence of convection flows).

In the overdamped regime, the sum of optical and Brownian forces acting on a particle is balanced by the drag force $F_{\mathrm{d}}$, which is proportional to the observed particle's velocity $v$. To remove the contribution of drag forces induced by macroscopic surface flows,
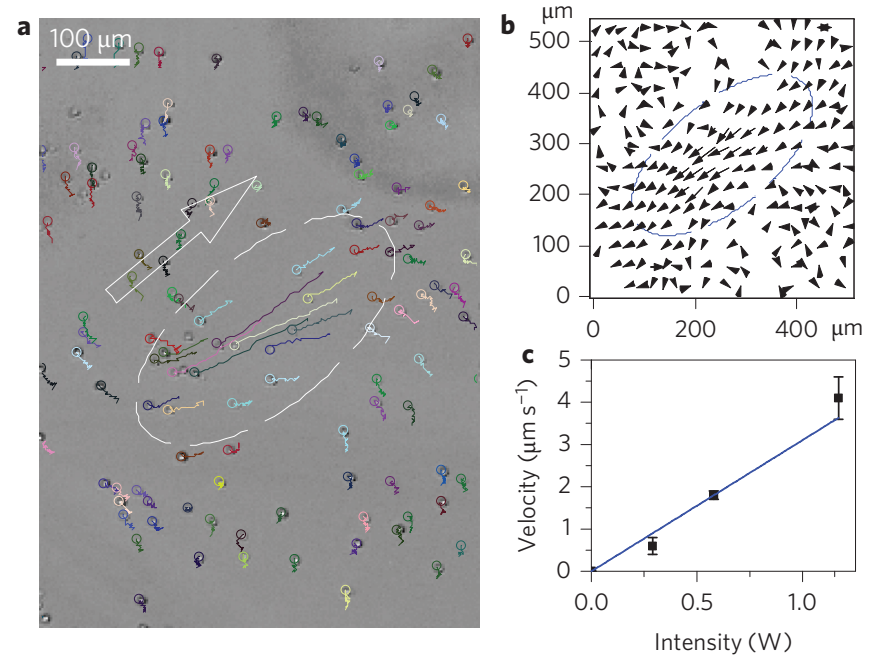

Figure 2 | Pulling action on particles bound to a water-air interface. a, Trajectories of oil drops in the field of view of a camera during the first $45 \mathrm{~s}$ of illumination with $p$-polarized light. The ends of trajectories are indicated by small circles and the dashed ellipse denotes the 1/e contour line of the illumination spot. The arrow indicates the direction of propagation of the incident beam (Supplementary Movie S1). b, Average velocities (due to optical forces acting along the interface) distribution at different locations relative to the illumination spot. The length of the arrows is proportional to the average speed, while their orientations indicate the local direction of movement after the contribution from macroscopic surface flows has been subtracted (see text). c, Average velocity of oil drops near the beam centre as a function of laser input power. Error bars indicate the standard deviation of the velocities distribution and the line is the linear fit to the data. 

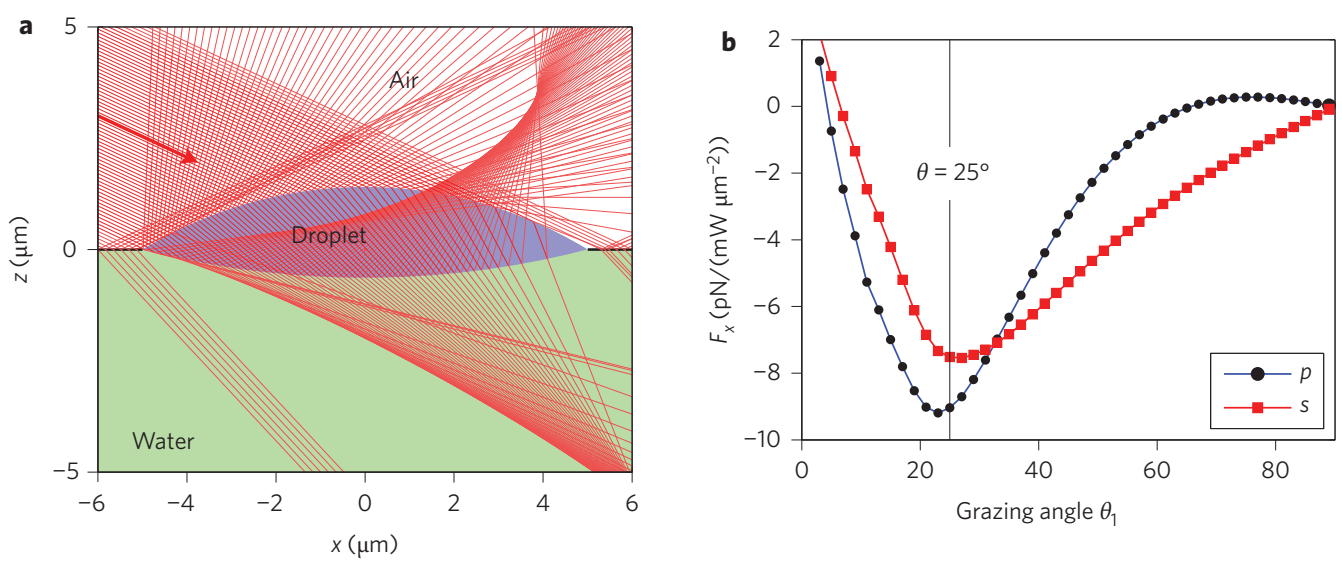

Figure 3 | Optical force exerted on a lens-like oil droplet at a water-air interface. a, Example of ray tracing for a floating lenslet-shaped particle. The crosssection of the lenslet is shown and only a very low ray density is displayed for clarity. $\mathbf{b}$. Optical force acting along the interface on a lens-like oil drop $10 \mu \mathrm{m}$ in diameter as a function of grazing angle $\theta_{1}$ for $s$ - and $p$-polarized incident waves, as indicated.

we subtracted the average velocity of the particles moving outside the illumination spot from the velocities determined for particles moving inside the spot. The corrected distribution of velocities in the case of $p$-polarized illumination is shown in Fig. $2 \mathrm{~b}$ and, as can be seen, it clearly demonstrates a pronounced movement against the direction of beam propagation. Note also that, because the illumination spot is rather large, the magnitude of the optical gradient forces is insignificant. These forces do not noticeably affect the recorded trajectories, as demonstrated in Fig. 2b, where one can see particles moving freely against the action of a potential gradient force, that is, from the centre of the beam down and left. A distribution of velocities similar to the one in Fig. 2b was also observed for the case of $s$-polarized illumination (not shown). Additional results illustrating the movement of different types of droplets are presented in Supplementary Section S3 (see also Supplementary Movie S2).

To study the dependence of the speed of induced movement of droplets on intensity, we performed several experiments where the illumination power was varied from $0.3 \mathrm{~W}$ to $1.2 \mathrm{~W}$. The results of velocity reconstruction are shown in Fig. 2c, where the linear dependence between these two parameters can be clearly seen.

The drag coefficient $b=F_{\mathrm{d}} / v$ at the surface of the water can be assessed independently by observing the Brownian motion of the oil drops in the absence of optical excitation (Supplementary Section S5). In the planar geometry of interest, the mean square displacement is related to the drag constant as $\left\langle r^{2}\right\rangle=4 k_{\mathrm{B}} T t / b$, where $k_{\mathrm{B}}$ is the Boltzmann constant and $T$ is the thermodynamic temperature. Using the measured drag coefficient $b=(2.3 \pm 0.1) \times 10^{-8} \mathrm{~N} \mathrm{~s} \mathrm{~m}^{-1}$ and the dependence between the observed velocity and irradiance shown in Fig. 2c, one can establish quantitatively the relative strength of the observed component of the negative force

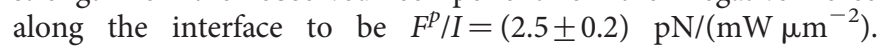
Performing a similar analysis for the $s$-polarized excitation, we

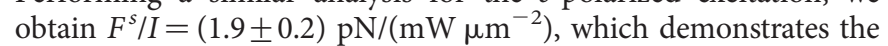
polarization dependence of the observed force.

To compare the experimental results with the predictions of, for instance, Minkowski's theory, one requires a correct description of the shape of the oil drops. In our experiment the oil drops were shaped as spherical lenses ${ }^{25}$ with the surface radii defined by surface tension coefficients (see Supplementary Section S4 for details). Although drag and electromagnetic forces may alter the shapes of soft dielectric interfaces ${ }^{16,24}$, the surface tension forces in our experiment are much larger (Supplementary Section S4) and do not allow significant deformation to the shape of the oil drops ${ }^{26,27}$. Using a ray-tracing method (Fig. 3a) as described in the Methods and Supplementary Section S1, we calculated the forces acting on droplets with the specific shape used in our experiments. Relating the momenta of the incident, reflected and transmitted rays within the Minkowski formalism, we obtained the optical forces for $p$ - and $s$-polarized incident waves as shown in Fig. 3b. As can be seen from the schematic illustration in Fig. 1a, this force is determined by the balance of three contributions to the total momentum, $\Delta \mathbf{p}=\mathbf{p}_{\mathrm{I}}-\mathbf{p}_{\mathrm{R}}-\mathbf{p}_{\mathrm{T}}$. The magnitudes of incident $\mathbf{p}_{\mathrm{I}}$ and reflected $\mathbf{p}_{\mathrm{R}}$ momenta in air are well defined. The apparent factor of four difference between the force measured experimentally and the value estimated numerically can be explained by a small variation of just $8 \%$ in the magnitude of the momentum in the medium $\mathbf{p}_{\mathrm{T}}$. Such deviation may be caused by several factors that were not taken into account in the model. First, the non-uniform electromagnetic field at the surface of the liquid oil drops can excite localized hydrodynamic flows that can affect the motion of the droplets. Second, the forces acting on closely spaced particles, often present in our experiment, could be different from that on a single particle, as considered in numerical estimations. Finally, there is also the possibility that interfacial phenomena (such as induced surface and interfacial charges reacting to the electric field of the incident beam ${ }^{28}$ ) in multiphase soft matter may require refinements of the microscopic theories of optical momentum exchange and the associated mechanical action. However, all these influences are just perturbations to the major contribution to the negative force created by the amplification of the momentum in water.

In summary, we have demonstrated that gradientless optical fields can act as 'tractor beams' along a properly chosen interface of two materials with different refractive indices. Based on the conservation of linear momentum, negative force can be generated without restrictions on the beam's shape, polarization, phase and so on. We note that optical forces and momentum exchange are usually discussed for objects entirely embedded in homogeneous media. The current experiment provides new insights for understanding surface effects in momentum exchange and can also assist in developing and evaluating microscopic theories of optical forces in multiphase systems and in soft bodies. This work also raises several other provocative questions. For example, could there be any specific coupling between electromagnetic and material energy-momentum tensors driven by the angular momentum conservation? This question is very interesting because light caries both angular and spin momenta, and Hall-type effects could, in principle, generate non-trivial stresses at soft interfaces.

Interestingly, the effect described in this Letter does not rely on any specific coherence properties of the optical field. Indeed, it can be achieved even with incoherent light. The amplification of the forward scattering momentum is due solely to the presence of 
an interface between low- and high-refractive-index media. The phenomenology discussed here could be translated easily to other wavelength domains. In contrast to another recent 'tractor beam' demonstration ${ }^{8}$, the experiment presented here is easy scalable for arbitrarily large objects and displacements. Also, the condition for objects to have specific shapes or to be highly scattering ${ }^{7}$ is not required. The simple scheme and the robust experimental implementation may find a plethora of applications in developing novel micromanipulation approaches and for studying interfacial phenomena.

\section{Materials and methods}

Experiment. Micrometre-sized floating liquid lenses were prepared according to the following procedure. Pure dodecane $(99 \%$, refractive index $n=1.421-1.423$, Acros Organics) was dissolved in pentane (anhydrous, $>99 \%$ from Sigma Aldrich) in the ratio 1:3,000 by volume. Small drops of the solution $(\sim 0.2 \mu \mathrm{l})$ were carefully released from a $10 \mu \mathrm{l}$ syringe (World Precision Instruments) onto the surface of water (ultrapure water, $24.7 \mathrm{M} \Omega \mathrm{cm}$ resistivity, $<5 \mathrm{ppb}$ total organic carbon) contained in a transparent plastic cuvette with a diameter of $3.8 \mathrm{~cm}$. The pentane evaporated rapidly, spontaneously forming oil droplets of dodecane $(\sim 10 \mu \mathrm{m})$ on the surface. Because the water and oils are immiscible, the oil drops on the water surface were stable for an extended period of time. The plastic cuvette was covered by a glass coverslip to prevent excessive drop movement due to air currents. The floating oil droplets were illuminated by a linearly polarized monochromatic light from a Nd:YVO ${ }_{4}$ laser (wavelength, $532 \mathrm{~nm}$; Millennia Vs, Spectra-Physics Lasers) The collimated laser beam with polarization controllable by a halfwave plate was focused by a long-focal-length biconvex lens $(f=500 \mathrm{~mm})$ and then directed onto the water surface. Laser power attenuation for a beam passing through optical components was properly taken into account. The water surface was also illuminated in transmission by an incoherent red light-emitting diode light source. The motion of oil drops at the water surface was imaged by a Zeiss microscope with a $\times 4$ objective (NA 0.10, Edmund) and recorded by a CCD camera (Andor iXon) with a frame rate of 0.67 f.p.s. A $532 \mathrm{~nm}$ notch filter (OD4 blocking, from Edmund) was used to remove the residual laser light scattered onto the microscope objective. The position and size of the laser spot on the surface were visualized by removing the notch filter.

Particle tracking. The determination of particle positions in individual frames (pretracking) was carried out with a homemade MATLAB code. Linking particles from different frames into trajectories (tracking) was performed with MATLAB code developed in M. Kilfoil's laboratory ${ }^{29}$.

Ray-tracing calculations. Calculation of optical forces was performed with a raytracing technique. The dimensions of the particles used in the experiment $(\sim 10 \mu \mathrm{m})$ were large enough compared to the wavelength of the incident light $(0.532 \mu \mathrm{m})$ for this approach to be valid with a high degree of accuracy. The adequate accuracy of the ray-tracing method in terms of calculation of optical forces was confirmed by comparing the results for two-dimensional objects of similar cross-sectional sizes with the results obtained with a finite-difference time-domain method. In the ray-tracing calculations, an incident light beam is regarded as a series of $N$ optical rays. In our calculation, $N$ was set to 2,500 , producing a relative error of $\sim 2 \%$ in the force calculation both for $p$ and $s$ polarization. This number of rays was found to be optimal to reduce the computation time and provide good convergence. Each ray carries $m$ photons with a momentum of $m p$ and energy of $m E_{0}$. Here $p=n(\hbar \omega / c)$ (according to Minkowski's formula) and $E_{0}=\hbar \omega$ are, respectively, the momentum and energy carried by a single photon in a material with refractive index of $n$. We calculated the directions and intensities (the number of photons) of the reflection and transmission rays when the ray encountered a sudden change in refractive index using the Fresnel formulae and vectorial Snell's law. The momentum change corresponding to each ray could be calculated. The total momentum changes of all rays could thus be obtained by tracing them until they left the system or until their intensity fell below a critical value. By summing the individual momentum changes for all rays, the total change in momentum could be determined and, in turn, the recoil force exerted on the scatter established.

Received 19 March 2013; accepted 28 June 2013; published online 11 August 2013

\section{References}

1. Ruffner, D. B. \& Grier, D. G. Optical conveyors: a class of active tractor beams. Phys. Rev. Lett. 109, 163903 (2012).

2. Chen, J., Ng, J., Lin, Z. \& Chan, C. T. Optical pulling force. Nature Photon. 5, 531-534 (2011).

3. Novitsky, A., Qiu, C.-W. \& Wang, H. Single gradientless light beam drags particles as tractor beams. Phys. Rev. Lett. 107, 203601 (2011).

4. Mizrahi, A. \& Fainman, Y. Negative radiation pressure on gain medium structures. Opt. Lett. 35, 3405-3407 (2010).
5. Salandrino, A. \& Christodoulides, D. N. Reverse optical forces in negative index dielectric waveguide arrays. Opt. Lett. 36, 3103-3105 (2011).

6. Nieto-Vesperinas, M., Sáenz, J. J., Gómez-Medina, R. \& Chantada, L. Optical forces on small magnetodielectric particles. Opt. Express 18, 11428-11443 (2010).

7. Sukhov, S. \& Dogariu, A. Negative nonconservative forces: optical 'tractor beams' for arbitrary objects. Phys. Rev. Lett. 107, 203602 (2011).

8. Brzobohatý, O. et al. Experimental demonstration of optical transport, sorting and self-arrangement using a 'tractor beam'. Nature Photon. 7, 123-127 (2013).

9. Dogariu, A., Sukhov, S. \& Saenz, J. J. Optically induced 'negative forces'. Nature Photon. 7, 24-27 (2013).

10. Ashkin, A. Acceleration and trapping of particles by radiation pressure. Phys. Rev. Lett. 24, 156-159 (1970).

11. Ashkin, A., Dziedzic, J. M., Bjorkholm, J. E. \& Chu, S. Observation of a single-beam gradient force optical trap for dielectric particles. Opt. Lett. 11, 288-290 (1986).

12. Bowman, R. W. \& Padgett, M. J. Optical trapping and binding. Rep. Prog. Phys. 76, 026401 (2013)

13. Li, M., Pernice, W. H. P. \& Tang, H. X. Tunable bipolar optical interactions between guided lightwaves. Nature Photon. 3, 464-468 (2009).

14. Wiederhecker, G. S., Chen, L., Gondarenko, A. \& Lipson, M. Controlling photonic structures using optical forces. Nature 462, 633-636 (2009).

15. Pfeifer, R. N. C., Nieminen, T. A., Heckenberg, N. R. \& Rubinsztein-Dunlop, H Colloquium: momentum of an electromagnetic wave in dielectric media. Rev. Mod. Phys. 79, 1197-1216 (2007).

16. Milonni, P. W. \& Boyd, R. W. Momentum of light in a dielectric medium. Adv. Opt. Photon. 2, 519-553 (2010).

17. Møller, C. The Theory of Relativity 2nd edn (Oxford Univ. Press, 1972).

18. Balazs, N. L. The energy-momentum tensor of the electromagnetic field inside matter. Phys. Rev. 91, 408-411 (1953).

19. Brevik, I. Experiments in phenomenological electrodynamics and the electromagnetic energy-momentum tensor. Phys. Rep. 52, 133-201 (1979).

20. Mikura, Z. Variational formulation of the electrodynamics of fluids and its application to the radiation pressure problem. Phys. Rev. A 13, 2265-2275 (1976).

21. Gordon, P. Radiation forces and momenta in dielectric media. Phys. Rev. A 8, 14-21 (1973).

22. Jones, R. V. \& Richards, J. C. S. The pressure of radiation in a refracting medium. Proc. R. Soc. Lond. A 221, 480-498 (1954).

23. Jones, R. V. \& Leslie, B. The measurement of optical radiation pressure in dispersive media. Proc. R. Soc. Lond. A 360, 347-363 (1978).

24. Ashkin, A. \& Dziedzic, J. M. Radiation pressure on a free liquid surface. Phys. Rev. Lett. 30, 139-142 (1973).

25. De Gennes, P-G., Brochard-Wart, F. \& Quéré, D. Capillarity and Wetting Phenomena: Drops, Bubbles, Pearls, Waves (Springer, 2003).

26. Pieranski, P. Two-dimensional interfacial colloidal crystals. Phys. Rev. Lett. 45, 569-572 (1980).

27. Radoev, B., Nedjalkov, M. \& Djakovich, V. Brownian motion at liquid-gas interfaces. 1. Diffusion coefficients of macroparticles at pure interfaces. Langmuir 8, 2962-2965 (1992).

28. Mansuripur, M. Radiation pressure and the linear momentum of the electromagnetic field. Opt. Express 12, 5375-5401 (2004).

29. Gao, Y. \& Kilfoil, M. L. Accurate detection and complete tracking of large populations of features in three dimensions. Opt. Express 17, 4685-4704 (2009).

\section{Acknowledgements}

This work was partially supported by the National Science Foundation (grant no. 1159530) and the Air Force Office of Scientific Research (no. FA9550-10-1-0190). C.-W.Q. acknowledges support from the National University of Singapore (grant no. R-263-000678-133). The authors thank J. Czarnecki for discussions regarding the properties of surface-bound water-oil emulsions and F.E. Hernandez for assistance with chemical preparation.

\section{Author contributions}

V.K., W.D., S.S., C.-W.Q. and A.D. designed the experiments, V.K. performed the experiments, W.D. and C.-W.Q. performed the theoretical simulations, S.S., C.-W.Q. and A.D. wrote the paper, C.-W.Q. and A.D. conceived the idea, and all authors contributed analysis tools.

\section{Additional information}

Supplementary information is available in the online version of the paper. Reprints and permissions information is available online at www.nature.com/reprints. Correspondence and requests for materials should be addressed to C.-W.Q. and A.D.

\section{Competing financial interests}

The authors declare no competing financial interests. 